\title{
Kummer Strikes Back: New DH Speed Records
}

\author{
Daniel J. Bernstein ${ }^{1,2}$, Chitchanok Chuengsatiansup ${ }^{2}$, Tanja Lange ${ }^{2}$, \\ and Peter Schwabe ${ }^{3}$ \\ 1 Department of Computer Science, University of Illinois at Chicago \\ Chicago, IL 60607-7045, USA \\ djb@cr.yp.to \\ 2 Department of Mathematics and Computer Science \\ Technische Universiteit Eindhoven \\ P.O. Box 513, 5600 MB Eindhoven, The Netherlands \\ c.chuengsatiansup@tue.nl, tanja@hyperelliptic.org \\ 3 Radboud University Nijmegen, Digital Security Group \\ P.O. Box 9010, 6500 GL Nijmegen, The Netherlands \\ peter@cryptojedi.org
}

\begin{abstract}
This paper sets new speed records for high-security constanttime variable-base-point Diffie-Hellman software: 305395 Cortex-A8-slow cycles; 273349 Cortex-A8-fast cycles; 88916 Sandy Bridge cycles; 88448 Ivy Bridge cycles; 54389 Haswell cycles. There are no higher speeds in the literature for any of these platforms.

The new speeds rely on a synergy between (1) state-of-the-art formulas for genus-2 hyperelliptic curves and (2) a modern trend towards vectorization in CPUs. The paper introduces several new techniques for efficient vectorization of Kummer-surface computations.
\end{abstract}

Keywords: performance, Diffie-Hellman, hyperelliptic curves, Kummer surfaces, vectorization.

\section{Introduction}

The Eurocrypt 2013 paper "Fast cryptography in genus 2" by Bos, Costello, Hisil, and Lauter [17] reported 117000 cycles on Intel's Ivy Bridge microarchitecture for high-security constant-time scalar multiplication on a genus-2 Kummer surface. The eBACS site for publicly verifiable benchmarks [13] confirms 119032 "cycles to compute a shared secret" (quartiles: 118904 and 119232) for the kumfp127g software from [17] measured on a single core of h9ivy, a 2012 Intel Core i5-3210M running at $2.5 \mathrm{GHz}$. The software is not much slower on Intel's previous microarchitecture, Sandy Bridge: eBACS reports 122716 cycles (quartiles: 122576 and 122836) for kumfp127g on h6sandy, a 2011 Intel Core i3-2310M running at $2.1 \mathrm{GHz}$. (The quartiles demonstrate that rounding to a

This work was supported by the National Science Foundation under grant 1018836 and by the Netherlands Organisation for Scientific Research (NWO) under grants 639.073.005, 613.001.011, and through the Veni 2013 project 13114. Permanent ID of this document: 1c5c0ead2524267af6b4f6d9114f10f0. Date: 2014.09.25.

P. Sarkar and T. Iwata (Eds.): ASIACRYPT 2014, PART I, LNCS 8873, pp. 317-337, 2014.

(C) International Association for Cryptologic Research 2014 
multiple of 1000 cycles, as in [17], loses statistically significant information; we follow eBACS in reporting medians of exact cycle counts.)

The paper reported that this was a "new software speed record" ("breaking the $120 \mathrm{k}$ cycle barrier") compared to "all previous genus 1 and genus 2 implementations" of high-security constant-time scalar multiplication. Obviously the genus-2 cycle counts shown above are better than the (unverified) claim of 137000 Sandy Bridge cycles by Longa and Sica in [40] (Asiacrypt 2012) for constanttime elliptic-curve scalar multiplication; the (unverified) claim of 153000 Sandy Bridge cycles by Hamburg in [34] for constant-time elliptic-curve scalar multiplication; the 182708 cycles reported by eBACS on h9ivy for curve25519, a constant-time implementation by Bernstein, Duif, Lange, Schwabe, and Yang [11] (CHES 2011) of Bernstein's Curve25519 elliptic curve [9]; and the 194036 cycles reported by eBACS on h6sandy for curve25519.

One might conclude from these figures that genus- 2 hyperelliptic-curve cryptography (HECC) solidly outperforms elliptic-curve cryptography (ECC). However, two newer papers claim better speeds for ECC, and a closer look reveals a strong argument that HECC should have trouble competing with ECC.

The first paper, [44] by Oliveira, López, Aranha, and Rodríguez-Henríquez (CHES 2013 best-paper award), is the new speed leader in eBACS for nonconstant-time scalar multiplication; the paper reports a new Sandy Bridge speed record of 69500 cycles. Much more interesting for us is that the paper claims 114800 Sandy Bridge cycles for constant-time scalar multiplication, beating [17]. eBACS reports 119904 cycles, but this is still faster than [17].

The second paper, [24] by Faz-Hernández, Longa, and Sánchez, claims 92000 Ivy Bridge cycles or 96000 Sandy Bridge cycles for constant-time scalar multiplication; a July 2014 update of the paper claims 89000 Ivy Bridge cycles or 92000 Sandy Bridge cycles. These claims are not publicly verifiable, but if they are even close to correct then they are faster than [17].

Both of these new papers, like [40], rely heavily on curve endomorphisms to eliminate many doublings, as proposed by Gallant, Lambert, and Vanstone [27] (Crypto 2001), patented by the same authors, and expanded by Galbraith, Lin, and Scott [26] (Eurocrypt 2009). Specifically, [44] uses a GLS curve over a binary field to eliminate $50 \%$ of the doublings, while also taking advantage of Intel's new pclmulqdq instruction to multiply binary polynomials; [24] uses a GLV+GLS curve over a prime field to eliminate $75 \%$ of the doublings.

One can also use the GLV and GLS ideas in genus 2, as explored by Bos, Costello, Hisil, and Lauter starting in [17] and continuing in [18] (CHES 2013). However, the best GLV/GLS speed reported in [18], 92000 Ivy Bridge cycles, provides only $2^{105}$ security and is not constant time. This is less impressive than the 119032 cycles from [17] for constant-time DH at a $2^{125}$ security level, and less impressive than the reports in [44] and [24].

The underlying problem for HECC is easy to explain. All known HECC addition formulas are considerably slower than the state-of-the-art ECC addition formulas at the same security level. Almost all of the HECC options explored in 
[17] are bottlenecked by additions, so they were doomed from the outset, clearly incapable of beating ECC.

The one exception is that HECC provides an extremely fast ladder (see Section 2), built from extremely fast differential additions and doublings, considerably faster than the Montgomery ladder frequently used for ECC. This is why [17] was able to set DH speed records.

Unfortunately, differential additions do not allow arbitrary addition chains. Differential additions are incompatible with standard techniques for removing most or all doublings from fixed-base-point single-scalar multiplication, and with standard techniques for removing many doublings from multi-scalar multiplication. As a consequence, differential additions are incompatible with the GLV+GLS approach mentioned above for removing many doublings from singlescalar multiplication. This is why the DH speeds from [17] were quickly superseded by DH speeds using GLV+GLS. A recent paper [22] (Eurocrypt 2014) by Costello, Hisil, and Smith shows feasibility of combining differential additions and use of endomorphisms but reports 145000 Ivy Bridge cycles for constanttime software, much slower than the papers mentioned above.

1.1. Contributions of This Paper. We show that HECC has an important compensating advantage, and we exploit this advantage to achieve new DH speed records. The advantage is that we are able to heavily vectorize the HECC ladder.

CPUs are evolving towards larger and larger vector units. A low-cost lowpower ARM Cortex-A8 CPU core contains a 128-bit vector unit that every two cycles can compute two vector additions, each producing four sums of 32-bit integers, or one vector multiply-add, producing two results of the form $a b+c$ where $a, b$ are 32-bit integers and $c$ is a 64-bit integer. Every cycle a Sandy Bridge CPU core can compute a 256-bit vector floating-point addition, producing four double-precision sums, and at the same time a 256-bit vector floating-point multiplication, producing four double-precision products. A new Intel Haswell CPU core can carry out two 256-bit vector multiply-add instructions every cycle. Intel has announced future support for 512-bit vectors ("AVX-512").

Vectorization has an obvious attraction for a chip manufacturer: the costs of decoding an instruction are amortized across many arithmetic operations. The challenge for the algorithm designer is to efficiently vectorize higher-level computations so that the available circuitry is performing useful work during these computations rather than sitting idle. What we show here is how to fit HECC with surprisingly small overhead into commonly available vector units. This poses several algorithmic challenges, notably to minimize the permutations required for the Hadamard transform (see Section 4). We claim broad applicability of our techniques to modern CPUs, and to illustrate this we analyze all three of the microarchitectures mentioned in the previous paragraph.

Beware that different microarchitectures often have quite different performance. A paper that advertises a "better" algorithmic idea by reporting new record cycle counts on a new microarchitecture, not considered in the previous literature, might actually be reporting an idea that loses performance on all microarchitectures. We instead emphasize HECC performance on the widely 
deployed Sandy Bridge microarchitecture, since Sandy Bridge was shared as a target by the recent ECC speed-record papers listed above. We have now set a new Sandy Bridge DH speed record, demonstrating the value of vectorized HECC. We have also set DH speed records for Ivy Bridge, Haswell, and CortexA8.

1.2. Constant Time: Importance and Difficulty. See full version of this paper online at https://eprint.iacr.org/2014/134.

1.3. Performance Results. eBACS shows that on a single core of h6sandy our DH software ("kummer") uses just 88916 Sandy Bridge cycles (quartiles: 88868 and 89184 ). On a single core of h9ivy our software uses 88448 cycles (quartiles: 88424 and 88476). On a single core of titan0, an Intel Xeon E3-1275 V3 (Haswell), our software uses 54389 cycles (quartiles: 54341 and 54454). On h7beagle, a TI Sitara AM3359 (Cortex-A8-slow), our software uses 305395 cycles (quartiles: 305380 and 305413). On h4mx515e, a Freescale i.MX515 (CortexA8-fast), our software uses 273349 cycles (quartiles: 273337 and 273387).

1.4. Cycle-Count Comparison. Table 1.5 summarizes reported high-security DH speeds for Cortex-A8, Sandy Bridge, Ivy Bridge, and Haswell.

This table is limited to software that claims to be constant time, and that claims a security level close to $2^{128}$. This is the reason that the table does not include, e.g., the 767000 Cortex-A8 cycles and 108000 Ivy Bridge cycles claimed in [18] for constant-time scalar multiplication on a Kummer surface; the authors claim only 103 bits of security for that surface. This is also the reason that the table does not include, e.g., the 69500 Sandy Bridge cycles claimed in [44] for non-constant-time scalar multiplication.

The table does not attempt to report whether the listed cycle counts are from software that actually meets the above security requirements. In some cases inspection of the software has shown that the security requirements are violated; see Section 1.2. "Open" means that the software is reported to be open source, allowing third-party inspection.

Our speeds, on the same platform targeted in [17], solidly beat the HECC speeds from [17]. Our speeds also solidly beat the Cortex-A8, Sandy Bridge, and Ivy Bridge speeds from all available ECC software, including [11], [15], [22], and [44]; solidly beat the speeds claimed in [34] and [40]; and are even faster than the July 2014 Sandy Bridge/Ivy Bridge DH record claimed in [24], namely 92000/89000 cycles using unpublished software for GLV+GLS ECC. For Haswell, despite Haswell's exceptionally fast binary-field multiplier, our speeds beat the 55595 cycles from [44] for a GLS curve over a binary field. We set our new speed records using an HECC ladder that is conceptually much simpler than GLV and GLS, avoiding all the complications of scalar-dependent precomputations, lattice size issues, multi-scalar addition chains, endomorphism-rho security analysis, Weil-descent security analysis, and patents. 
Table 1.5. Reported high-security DH speeds for Cortex-A8, Sandy Bridge, Ivy Bridge, and Haswell. Cycle counts from eBACS are for curve25519, kumfp127g, gls254prot, and our kummer on h7beagle (Cortex-A8-slow), h4mx515e (Cortex-A8-fast), h6sandy (Sandy Bridge), h9ivy (Ivy Bridge), and titano (Haswell). Cycle counts not from SUPERCOP are marked "?". ECC has $g=1$; genus-2 HECC has $g=2$. See text for security requirements.

\begin{tabular}{|c|c|c|c|c|c|c|}
\hline $\operatorname{arch}$ & cycles & lad & open & $g$ field & \multicolumn{2}{|c|}{ source of software } \\
\hline A8-slow & 497389 & yes & yes & \begin{tabular}{l|l}
1 & $2^{255}-19$
\end{tabular} & {$[15]$} & CHES 2012 \\
\hline A8-slow & 305395 & yes & yes & \begin{tabular}{l|l}
2 & $2^{127}-1$ \\
\end{tabular} & \multicolumn{2}{|c|}{ new (this paper) } \\
\hline A8-fast & 460200 & yes & yes & \begin{tabular}{|l|l|}
1 & $2^{255}-19$
\end{tabular} & {$[15]$} & CHES 2012 \\
\hline A8-fast & 273349 & yes & yes & $2 \mid 2^{127}-1$ & \multicolumn{2}{|c|}{ new (this paper) } \\
\hline Sandy & 194036 & yes & yes & \begin{tabular}{|l|l}
1 & $2^{255}-19$
\end{tabular} & {$[11]$} & CHES 2011 \\
\hline Sandy & $153000 ?$ & yes & no & $12^{252}-2^{232}-1$ & {$[34]$} & \\
\hline Sandy & $137000 ?$ & no & no & $1\left(2^{127}-5997\right)^{2}$ & {$[40]$} & Asiacrypt 2012 \\
\hline Sandy & 122716 & yes & yes & $2 \mid 2^{127}-1$ & {$[17]$} & Eurocrypt 2013 \\
\hline Sandy & 119904 & no & yes & $12^{254}$ & {$[44]$} & CHES 2013 \\
\hline Sandy & $96000 ?$ & no & no & $1\left(2^{127}-5997\right)^{2}$ & {$[24]$} & CT-RSA 2014 \\
\hline Sandy & $92000 ?$ & no & no & $1\left(2^{127}-5997\right)^{2}$ & {$[24]$} & July 2014 \\
\hline Sandy & 88916 & yes & yes & $2 \mid 2^{127}-1$ & \multicolumn{2}{|c|}{ new (this paper) } \\
\hline Ivy & 182708 & yes & yes & \begin{tabular}{l|l}
1 & $2^{255}-19$
\end{tabular} & {$[11]$} & CHES 2011 \\
\hline Ivy & $145000 ?$ & yes & yes & $1\left(2^{127}-1\right)^{2}$ & {$[22]$} & Eurocrypt 2014 \\
\hline Ivy & 119032 & yes & yes & $2 \mid 2^{127}-1$ & {$[17]$} & Eurocrypt 2013 \\
\hline Ivy & 114036 & no & yes & $12^{254}$ & {$[44]$} & CHES 2013 \\
\hline Ivy & $92000 ?$ & no & no & $1\left(2^{127}-5997\right)^{2}$ & {$[24]$} & CT-RSA 2014 \\
\hline Ivy & $89000 ?$ & no & no & $1\left(2^{127}-5997\right)^{2}$ & {$[24]$} & July 2014 \\
\hline Ivy & 88448 & yes & yes & $2 \mid 2^{127}-1$ & \multicolumn{2}{|c|}{ new (this paper) } \\
\hline Haswell & 145907 & yes & yes & \begin{tabular}{l|l}
1 & $2^{255}-19$
\end{tabular} & {$[11]$} & CHES 2011 \\
\hline Haswell & 100895 & yes & yes & $2 \mid 2^{127}-1$ & {$[17]$} & Eurocrypt 2013 \\
\hline Haswell & 55595 & no & yes & $12^{254}$ & {$[44]$} & CHES 2013 \\
\hline Haswell & 54389 & yes & yes & $2 \mid 2^{127}-1$ & \multicolumn{2}{|c|}{ new (this paper) } \\
\hline
\end{tabular}

\section{Fast Scalar Multiplication on the Kummer Surface}

This section reviews the smallest number of field operations known for genus-2 scalar multiplication. Sections 3 and 4 optimize the performance of those field operations using 4-way vector instructions.

Vectorization changes the interface between this section and subsequent sections. What we actually optimize is not individual field operations, but rather pairs of operations, pairs of pairs, etc., depending on the amount of vectorization available from the CPU. Our optimization also takes advantage of sequences of operations such as the output of a squaring being multiplied by a small constant. What matters in this section is therefore not merely the number of field multiplications, squarings, etc., but also the pattern of those operations.

2.1. Only 25 Multiplications. Almost thirty years ago Chudnovsky and Chudnovsky wrote a classic paper [21] optimizing scalar multiplication inside the elliptic-curve method of integer factorization. At the end of the paper they 


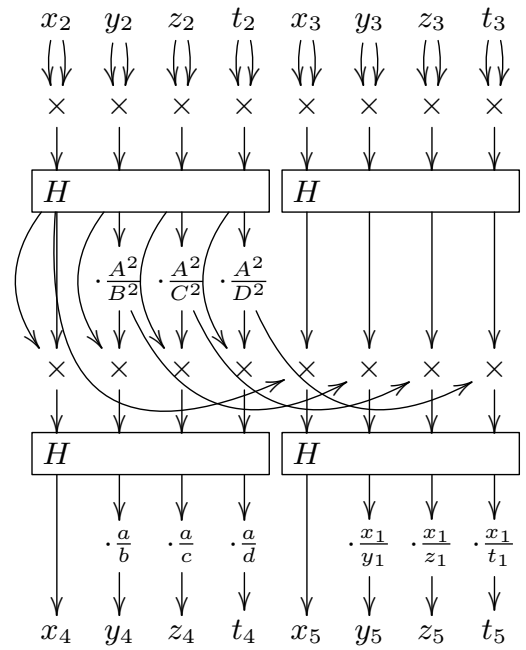

(a) $10 \mathbf{M}+9 \mathbf{S}+6 \mathbf{m}$ ladder formulas.

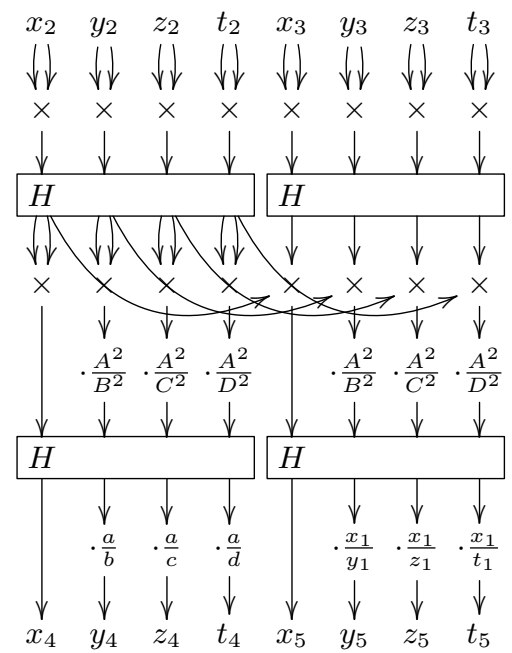

(b) $7 \mathbf{M}+12 \mathbf{S}+9 \mathbf{m}$ ladder formulas.

Fig. 2.2. Ladder formulas for the Kummer surface. Inputs are $X(Q-P)=\left(x_{1}\right.$ : $\left.y_{1}: z_{1}: t_{1}\right), X(P)=\left(x_{2}: y_{2}: z_{2}: t_{2}\right)$, and $X(Q)=\left(x_{3}: y_{3}: z_{3}: t_{3}\right)$; outputs are $X(2 P)=\left(x_{4}: y_{4}: z_{4}: t_{4}\right)$ and $X(P+Q)=\left(x_{5}: y_{5}: z_{5}: t_{5}\right)$. Formulas in (a) are from Gaudry [30]; diagrams are copied from Bernstein [10].

also considered the performance of scalar multiplication on Jacobian varieties of genus-2 hyperelliptic curves. After mentioning various options they gave some details of one option, namely scalar multiplication on a Kummer surface.

A Kummer surface is related to the Jacobian of a genus-2 hyperelliptic curve in the same way that $x$-coordinates are related to a Weierstrass elliptic curve. There is a standard rational map $X$ from the Jacobian to the Kummer surface; this map satisfies $X(P)=X(-P)$ for points $P$ on the Jacobian and is almost everywhere exactly 2-to-1. Addition on the Jacobian does not induce an operation on the Kummer surface (unless the number of points on the surface is extremely small), but scalar multiplication $P \mapsto n P$ on the Jacobian induces scalar multiplication $X(P) \mapsto X(n P)$ on the Kummer surface. Not every genus-2 hyperelliptic curve can have its Jacobian mapped to the standard type of Kummer surface over the base field, but a noticeable fraction of curves can; see [31].

Chudnovsky and Chudnovsky reported 14M for doubling a Kummer-surface point, where $\mathbf{M}$ is the cost of field multiplication; and $23 \mathbf{M}$ for "general addition", presumably differential addition, computing $X(Q+P)$ given $X(P), X(Q)$, $X(Q-P)$. They presented their formulas for doubling, commenting on a "pretty symmetry" in the formulas and on the number of multiplications that were actually squarings. They did not present their formulas for differential addition.

Two decades later, in [30], Gaudry reduced the total cost of differential addition and doubling, computing $X(2 P), X(Q+P)$ given $X(P), X(Q), X(Q-P)$, to $25 \mathbf{M}$, more precisely $16 \mathbf{M}+9 \mathbf{S}$, more precisely $10 \mathbf{M}+9 \mathbf{S}+6 \mathbf{m}$, where $\mathbf{S}$ is 


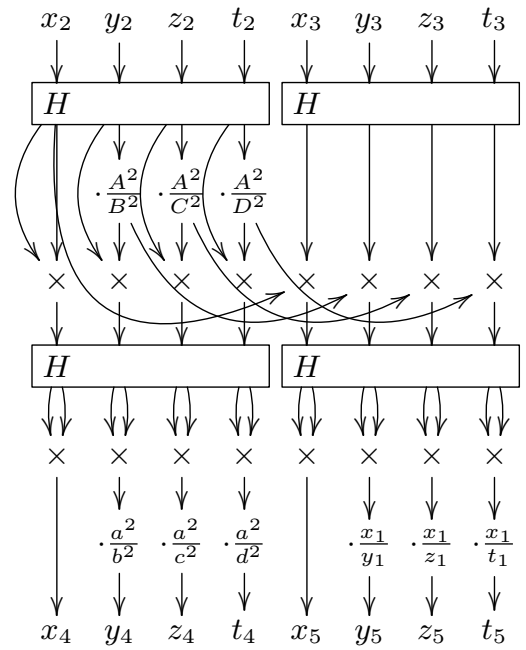

(a) $10 \mathbf{M}+9 \mathbf{S}+6 \mathbf{m}$ ladder formulas.

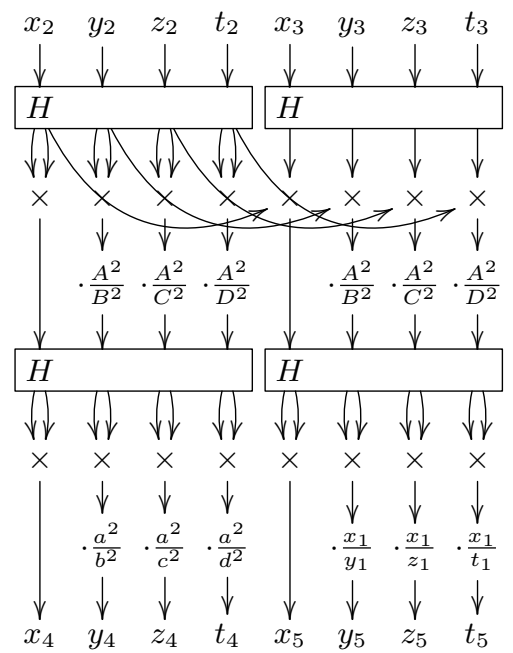

(b) $7 \mathbf{M}+12 \mathbf{S}+9 \mathbf{m}$ ladder formulas.

Fig. 2.4. Ladder formulas for the squared Kummer surface. Compare to Figure 2.2.

the cost of field squaring and $\mathbf{m}$ is the cost of multiplication by a curve constant. An $\ell$-bit scalar-multiplication ladder therefore costs just $10 \ell \mathbf{M}+9 \ell \mathbf{S}+6 \ell \mathbf{m}$.

Gaudry's formulas are shown in Figure 2.2(a). Each point on the Kummer surface is expressed projectively as four field elements $(x: y: z: t)$; one is free to replace $(x: y: z: t)$ with $(r x: r y: r z: r t)$ for any nonzero $r$. The " $H$ " boxes are Hadamard transforms, each using 4 additions and 4 subtractions; see Section 4 . The Kummer surface is parametrized by various constants $(a: b: c: d)$ and related constants $\left(A^{2}: B^{2}: C^{2}: D^{2}\right)=H\left(a^{2}: b^{2}: c^{2}: d^{2}\right)$. The doubling part of the diagram, from $\left(x_{2}: y_{2}: z_{2}: t_{2}\right)$ down to $\left(x_{4}: y_{4}: z_{4}: t_{4}\right)$, uses $3 \mathbf{M}+5 \mathbf{S}+6 \mathbf{m}$, matching the $14 \mathbf{M}$ reported by Chudnovsky and Chudnovsky; but the rest of the picture uses just $7 \mathbf{M}+4 \mathbf{S}$ extra, making remarkable reuse of the intermediate results of doubling. Figure $2.2(\mathrm{~b})$ replaces $10 \mathbf{M}+9 \mathbf{S}+6 \mathbf{m}$ with $7 \mathbf{M}+12 \mathbf{S}+9 \mathbf{m}$, as suggested by Bernstein in [10]; this saves time if $\mathbf{m}$ is smaller than the difference $\mathbf{M}-\mathbf{S}$.

\subsection{The Original Kummer Surface vs. The Squared Kummer Surface.}

Chudnovsky and Chudnovsky had actually used slightly different formulas for a slightly different surface, which we call the "squared Kummer surface". Each point $(x: y: z: t)$ on the original Kummer surface corresponds to a point $\left(x^{2}: y^{2}: z^{2}: t^{2}\right)$ on the squared Kummer surface. Figure 2.4 presents the equivalent of Gaudry's formulas for the squared Kummer surface, relabeling $\left(x^{2}: y^{2}: z^{2}: t^{2}\right)$ as $(x: y: z: t)$; the squarings at the top of Figure 2.2 have moved close to the bottom of Figure 2.4.

The number of field operations is the same either way, as stated in [10] with credit to André Augustyniak. However, the squared Kummer surface has a computational advantage over the original Kummer surface, as pointed out by 
Bernstein in [10]: constructing surfaces in which all of $a^{2}, b^{2}, c^{2}, d^{2}, A^{2}, B^{2}, C^{2}, D^{2}$ are small, producing fast multiplications by constants in Figure 2.4, is easier than constructing surfaces in which all of $a, b, c, d, A^{2}, B^{2}, C^{2}, D^{2}$ are small, producing fast multiplications by constants in Figure 2.2.

2.5. Preliminary Comparison to ECC. A Montgomery ladder step for ECC costs $5 \mathbf{M}+4 \mathbf{S}+1 \mathbf{m}$, while a ladder step on the Kummer surface costs $10 \mathbf{M}+9 \mathbf{S}+$ $6 \mathbf{m}$ or $7 \mathbf{M}+12 \mathbf{S}+9 \mathbf{m}$. Evidently ECC uses only about half as many operations. However, for security ECC needs primes around 256 bits (such as the convenient prime $\left.2^{255}-19\right)$, while the Kummer surface can use primes around 128 bits (such as the even more convenient prime $2^{127}-1$ ), and presumably this saves more than a factor of 2 .

Several years ago, in [10], Bernstein introduced 32-bit Intel Pentium M software for generic Kummer surfaces (i.e., $\mathbf{m}=\mathbf{M}$ ) taking about $10 \%$ fewer cycles than his Curve25519 software, which at the time was the speed leader for ECC. Gaudry, Houtmann, and Thomé, as reported in [32, comparison table], introduced 64-bit software for Curve25519 and for a Kummer surface; the second option was slightly faster on AMD Opteron K8 but the first option was slightly faster on Intel Core 2. It is not at all clear that one can reasonably extrapolate to today's CPUs.

Bernstein's cost analysis concluded that HECC could be as much as $1.5 \times$ faster than ECC on a Pentium M (cost 1355 vs. cost 1998 in [10, page 31]), depending on the exact size of the constants $a^{2}, b^{2}, c^{2}, d^{2}, A^{2}, B^{2}, C^{2}, D^{2}$. This motivated a systematic search through small constants to find a Kummer surface providing high security and high twist security. But this was more easily said than done: genus-2 point counting was much more expensive than elliptic-curve point counting.

2.6. The Gaudry-Schost Kummer Surface. Years later, after a 1000000CPU-hour computation relying on various algorithmic improvements to genus-2 point counting, Gaudry and Schost announced in [33] that they had found a secure Kummer surface $\left(a^{2}: b^{2}: c^{2}: d^{2}\right)=(11:-22:-19:-3)$ over $\mathbf{F}_{p}$ with $p=2^{127}-1$. This is exactly the surface that was used for the HECC speed records in [17]. We obtain even better speeds for the same surface.

Note that, as mentioned by Bos, Costello, Hisil, and Lauter in [17], the constants $\left(1: a^{2} / b^{2}: a^{2} / c^{2}: a^{2} / d^{2}\right)=(1:-1 / 2:-11 / 19:-11 / 3)$ in Figure 2.4 are projectively the same as $(-114: 57: 66: 418)$. The common factor 11 between $a^{2}=11$ and $b^{2}=-22$ helps keep these integers small. The constants $\left(1: A^{2} / B^{2}: A^{2} / C^{2}: A^{2} / D^{2}\right)=(1:-3:-33 / 17:-33 / 49)$ are projectively the same as $(-833: 2499: 1617: 561)$.

\section{Decomposing Field Multiplication}

The only operations in Figures 2.2 and 2.4 are the $H$ boxes, which we analyze in Section 4, and field multiplications, which we analyze in this section. Our goal here is to obtain the smallest possible number of CPU cycles for $\mathbf{M}, \mathbf{S}$, etc. modulo $p=2^{127}-1$. 
This prime has been considered before, for example in [8] and [10]. What is new here is fitting arithmetic modulo this prime, for the pattern of operations shown in Figure 2.4, into the vector abilities of modern CPUs. There are four obvious dimensions of vectorizability:

- Vectorizing across the "limbs" that represent a field element such as $x_{2}$. The most obvious problem with this approach is that, when $f$ is multiplied by $g$, each limb of $f$ needs to communicate with each limb of $g$ and each limb of output. A less obvious problem is that the optimal number of limbs is CPU-dependent and is usually nonzero modulo the vector length. Each of these problems poses a challenge in organizing and reshuffling data inside multiplications.

- Vectorizing across the four field elements that represent a point. All of the multiplications in Figure 2.4 are visually organized into 4-way vectors, except that in some cases the vectors have been scaled to create a multiplication by 1 . Even without vectorization, most of this scaling is undesirable for any surface with small $a^{2}, b^{2}, c^{2}, d^{2}$ : e.g., for the Gaudry-Schost surface we replace $\left(1: a^{2} / b^{2}: a^{2} / c^{2}: a^{2} / d^{2}\right)$ with $(-114: 57: 66: 418)$. The only remaining exception is the multiplication by 1 in $\left(1: x_{1} / y_{1}: x_{1} / z_{1}: x_{1} / t_{1}\right)$ where $X(Q-P)=\left(x_{1}: y_{1}: z_{1}: t_{1}\right)$. Vectorizing across the four field elements means that this multiplication costs $1 \mathbf{M}$, increasing the cost of a ladder step from $7 \mathbf{M}+12 \mathbf{S}+12 \mathbf{m}$ to $8 \mathbf{M}+12 \mathbf{S}+12 \mathbf{m}$.

- Vectorizing between doubling and differential addition. For example, in Figure 2.4(b), squarings are imperfectly paired with multiplications on the third line; multiplications by constants are perfectly paired with multiplications by the same constants on the fourth line; squarings are perfectly paired with squarings on the sixth line; and multiplications by constants are imperfectly paired with multiplications by inputs on the seventh line. There is some loss of efficiency in, e.g., pairing the squaring with the multiplication, since this prohibits using faster squaring methods.

- Vectorizing across a batch of independent scalar-multiplication inputs, in applications where a suitably sized batch is available. This is relatively straightforward but increases cache traffic, often to problematic levels. In this paper we focus on the traditional case of a single input.

The second dimension of vectorizability is, as far as we know, a unique feature of HECC, and one that we heavily exploit for high performance.

For comparison, one can try to vectorize the well-known Montgomery ladder for ECC [42] across the field elements that represent a point, but (1) this provides only two-way vectorization ( $x$ and $z$ ), not four-way vectorization; and (2) many of the resulting pairings are imperfect. The Montgomery ladder for Curve25519 was vectorized by Costigan and Schwabe in [23] for the Cell, and then by Bernstein and Schwabe in [15] for the Cortex-A8, but both of those vectorizations had substantially higher overhead than our new vectorization of the HECC ladder.

3.1. Sandy Bridge Floating-Point Units. The only fast multiplier available on Intel's 32-bit platforms for many years, from the original Pentium twenty 
years ago through the Pentium M, was the floating-point multiplier. This was exploited by Bernstein for cryptographic computations in [8], [9], etc.

The conventional wisdom is that this use of floating-point arithmetic was rendered obsolete by the advent of 64-bit platforms: in particular, Intel now provides a reasonably fast 64-bit integer multiplier. However, floating-point units have also become more powerful; evidently Intel sees many applications that rely critically upon fast floating-point arithmetic. We therefore revisit Bernstein's approach, with the added challenge of vectorization.

We next describe the relevant features of the Sandy Bridge; see [25] for more information. Our optimization of HECC for the Sandy Bridge occupies the rest of Sections 3 and 4 . The Ivy Bridge has the same features and should be expected to produce essentially identical performance for this type of code. The Haswell has important differences and is analyzed in Appendix B online; the Cortex-A8 is analyzed in Section 5 .

Each Sandy Bridge core has several 256-bit vector units operating in parallel on vectors of 4 double-precision floating-point numbers:

- "Port 0" handles one vector multiplication each cycle, with latency 5.

- Port 1 handles one vector addition each cycle, with latency 3.

- Port 5 handles one permutation instruction each cycle. The selection of permutation instructions is limited and is analyzed in detail in Section 4.

- Ports 2, 3, and 4 handle vector loads and stores, with latency 4 from L1 cache and latency 3 to L1 cache. Load/store throughput is limited in various ways, never exceeding one 256-bit load per cycle.

Recall that a double-precision floating-point number occupies 64 bits, including a sign bit, a power of 2 , and a "mantissa". Every integer between $-2^{53}$ and $2^{53}$ can be represented exactly as a double-precision floating-point number. More generally, every real number of the form $2^{e} i$, where $e$ is a small integer and $i$ is an integer between $-2^{53}$ and $2^{53}$, can be represented exactly as a double-precision floating-point number. The computations discussed here do not approach the lower or upper limits on $e$, so we do not review the details of the limits.

Our final software uses fewer multiplications than additions, and fewer permutations than multiplications. This does not mean that we were free to use extra multiplications and permutations: if multiplications and permutations are not finished quickly enough then the addition unit will sit idle waiting for input. In many cases, noted below, we have the flexibility to convert multiplications to additions, reducing latency; we found that in some cases this saved time despite the obvious addition bottleneck.

3.2. Optimizing $\mathbf{M}$ (Field Multiplication). We decompose an integer $f$ modulo $2^{127}-1$ into six floating-point limbs in (non-integer) radix $2^{127 / 6}$. This means that we write $f$ as $f_{0}+f_{1}+f_{2}+f_{3}+f_{4}+f_{5}$ where $f_{0}$ is a small multiple of $2^{0}, f_{1}$ is a small multiple of $2^{22}, f_{2}$ is a small multiple of $2^{43}, f_{3}$ is a small multiple of $2^{64}$, $f_{4}$ is a small multiple of $2^{85}$, and $f_{5}$ is a small multiple of $2^{106}$. (The exact meaning of "small" is defined by a rather tedious, but verifiable, collection of bounds on the floating-point numbers appearing in each step of the program. It should 
be obvious that a simpler definition of "small" would compromise efficiency; for example, $H$ cannot be efficient unless the bounds on $H$ intermediate results and outputs are allowed to be larger than the bounds on $H$ inputs.)

If $g$ is another integer similarly decomposed as $g_{0}+g_{1}+g_{2}+g_{3}+g_{4}+g_{5}$ then $f_{0} g_{0}$ is a multiple of $2^{0}, f_{0} g_{1}+f_{1} g_{0}$ is a multiple of $2^{22}, f_{0} g_{2}+f_{1} g_{1}+f_{2} g_{0}$ is a multiple of $2^{43}$, etc. Each of these sums is small enough to fit exactly in a double-precision floating-point number, and the total of these sums is exactly $f g$. What we actually compute are the sums

$$
\begin{aligned}
& h_{0}=f_{0} g_{0}+2{ }^{127} f_{1} g_{5}+2{ }^{127} f_{2} g_{4}+2{ }^{127} f_{3} g_{3}+2{ }^{127} f_{4} g_{2}+2{ }^{127} f_{5} g_{1} \text {, } \\
& h_{1}=f_{0} g_{1}+\quad f_{1} g_{0}+2{ }^{127} f_{2} g_{5}+2{ }^{127} f_{3} g_{4}+2{ }^{127} f_{4} g_{3}+2{ }^{127} f_{5} g_{2} \text {, } \\
& h_{2}=f_{0} g_{2}+f_{1} g_{1}+\quad f_{2} g_{0}+2^{127} f_{3} g_{5}+2^{127} f_{4} g_{4}+2{ }^{127} f_{5} g_{3}, \\
& h_{3}=f_{0} g_{3}+f_{1} g_{2}+f_{2} g_{1}+\quad f_{3} g_{0}+2^{127} f_{4} g_{5}+2^{127} f_{5} g_{4}, \\
& h_{4}=f_{0} g_{4}+f_{1} g_{3}+f_{2} g_{2}+f_{3} g_{1}+f_{4} g_{0}+2^{127} f_{5} g_{5}, \\
& h_{5}=f_{0} g_{5}+f_{1} g_{4}+f_{2} g_{3}+f_{3} g_{2}+f_{4} g_{1}+f_{5} g_{0},
\end{aligned}
$$

whose total $h$ is congruent to $f g$ modulo $2^{127}-1$.

There are 36 multiplications $f_{i} g_{j}$ here, and 30 additions. (This operation count does not include carries; we analyze carries below.) One can collect the multiplications by $2^{-127}$ into 5 multiplications such as $2^{-127}\left(f_{4} g_{5}+f_{5} g_{4}\right)$. We use another approach, precomputing $2^{-127} f_{1}, 2^{-127} f_{2}, 2^{-127} f_{3}, 2^{-127} f_{4}, 2^{-127} f_{5}$, for two reasons: first, this reduces the latency of each $h_{i}$ computation, giving us more flexibility in scheduling; second, this gives us an opportunity to share precomputations when the input $f$ is reused for another multiplication.

\subsection{Optimizing $\mathrm{S}$ (Field Squaring) and $\mathrm{m}$ (Constant Field Multipli-} cation). For $\mathbf{S}$, i.e., for $f=g$, we have

$$
\begin{array}{lll}
h_{0}=f_{0} f_{0}+\epsilon 2 f_{1} f_{5}+\epsilon 2 f_{2} f_{4}+\epsilon f_{3} f_{3}, & h_{1}=2 f_{0} f_{1}+\epsilon 2 f_{2} f_{5}+\epsilon 2 f_{3} f_{4}, \\
h_{2}=2 f_{0} f_{2}+f_{1} f_{1}+\epsilon 2 f_{3} f_{5}+\epsilon f_{4} f_{4}, & h_{3}=2 f_{0} f_{3}+2 f_{1} f_{2}+\epsilon 2 f_{4} f_{5}, \\
h_{4}=2 f_{0} f_{4}+2 f_{1} f_{3}+f_{2} f_{2}+\epsilon f_{5} f_{5}, & h_{5}=2 f_{0} f_{5}+2 f_{1} f_{4}+2 f_{2} f_{3}
\end{array}
$$

where $\epsilon=2^{-127}$. We precompute $2 f_{1}, 2 f_{2}, 2 f_{3}, 2 f_{4}, 2 f_{5}$ and $\epsilon f_{3}, \epsilon f_{4}, \epsilon f_{5}$; this costs 8 multiplications, where 5 of the multiplications can be freely replaced by additions. The rest of $\mathbf{S}$, after this precomputation, takes 21 multiplications and 15 additions, plus the cost of carries.

For $\mathbf{m}$ we have simply $h_{0}=c f_{0}, h_{1}=c f_{1}$, etc., costing 6 multiplications plus the cost of carries. This does not work for arbitrary field constants, but it does work for the small constants stated in Section 2.6.

3.4. Carries. The output limbs $h_{i}$ from $\mathbf{M}$ are too large to be used in a subsequent multiplication. We carry $h_{0} \rightarrow h_{1}$ by rounding $2^{-22} h_{0}$ to an integer $c_{0}$, adding $2^{22} c_{0}$ to $h_{1}$, and subtracting $2^{22} c_{0}$ from $h_{0}$. This takes 3 additions (the CPU has a rounding instruction, vroundpd, that costs just 1 addition) and 2 multiplications. The resulting $h_{0}$ is guaranteed to be between $-2^{21}$ and $2^{21}$.

We could similarly carry $h_{1} \rightarrow h_{2} \rightarrow h_{3} \rightarrow h_{4} \rightarrow h_{5}$, and carry $h_{5} \rightarrow h_{0}$ as follows: round $2^{-127} h_{5}$ to an integer $c_{5}$, add $c_{5}$ to $h_{0}$, and subtract $2^{127} c_{5}$ 
from $h_{5}$. One final carry $h_{0} \rightarrow h_{1}$, for a total of 7 carries $(21$ additions and 14 multiplications), would then guarantee that all of $h_{0}, h_{1}, h_{2}, h_{3}, h_{4}, h_{5}$ are small enough to be input to a subsequent multiplication.

The problem with this carry chain is that it has extremely high latency: 5 cycles for $2^{-22} h_{0}, 3$ more cycles for $c_{0}, 5$ more cycles for $2^{22} c_{0}$, and 3 more cycles to add to $h_{1}$, all repeated 7 times, for a total of 112 cycles, plus the latency of obtaining $h_{0}$ in the first place. The ladder step in Figure 2.4 has a serial chain of $H \rightarrow \mathbf{M} \rightarrow \mathbf{m} \rightarrow H \rightarrow \mathbf{S} \rightarrow \mathbf{M}$, for a total latency above 500 cycles, i.e., above 125500 cycles for a 251-bit ladder.

We do better in six ways. First, we use only 6 carries in $\mathbf{M}$ rather than 7 , if the output will be used only for $\mathbf{m}$. Even if the output $h_{0}$ is several bits larger than $2^{22}$, it will not overflow the small-constant multiplication, since our constants are all bounded by $2^{12}$.

Second, pushing the same idea further, we do these 6 carries in parallel. First we round in parallel to obtain $c_{0}, c_{1}, c_{2}, c_{3}, c_{4}, c_{5}$, then we subtract in parallel, then we add in parallel, allowing all of $h_{0}, h_{1}, h_{2}, h_{3}, h_{4}, h_{5}$ to end up several bits larger than they would have been with full carries.

Third, we also use 6 parallel carries for a multiplication that is an $\mathbf{m}$. There is no need for a chain, since the initial $h_{0}, h_{1}, h_{2}, h_{3}, h_{4}, h_{5}$ cannot be very large.

Fourth, we also use 6 parallel carries for each $\mathbf{S}$. This allows the $\mathbf{S}$ output to be somewhat larger than the input, but this still does not create overflows in the subsequent $\mathbf{M}$. At this point the only remaining block of 7 carries is in the $\mathbf{M}^{\mathbf{4}}$ by $\left(1: x_{1} / y_{1}: x_{1} / z_{1}: x_{1} / t_{1}\right)$, where $\mathbf{M}^{\mathbf{4}}$ means a vector of four field multiplications.

Fifth, for that $\mathbf{M}^{\mathbf{4}}$, we run two carry chains in parallel, carrying $h_{0} \rightarrow h_{1}$ and $h_{3} \rightarrow h_{4}$, then $h_{1} \rightarrow h_{2}$ and $h_{4} \rightarrow h_{5}$, then $h_{2} \rightarrow h_{3}$ and $h_{5} \rightarrow h_{0}$, then $h_{3} \rightarrow h_{4}$ and $h_{0} \rightarrow h_{1}$. This costs 8 carries rather than 7 but chops latency in half.

Finally, for that $\mathbf{M}^{\mathbf{4}}$, we use the carry approach from [8]: add the constant $\alpha_{22}=2^{22}\left(2^{52}+2^{51}\right)$ to $h_{0}$, and subtract $\alpha_{22}$ from the result, obtaining the closest multiple of $2^{22}$ to $h_{0}$; add this multiple to $h_{1}$ and subtract it from $h_{0}$. This costs 4 additions rather than 3 , but reduces carry latency from 16 to 9 , and also saves two multiplications.

\section{Permutations: Vectorizing the Hadamard Transform}

The Hadamard transform $H$ in Section 2 is defined as follows: $H(x, y, z, t)=$ $(x+y+z+t, x+y-z-t, x-y+z-t, x-y-z+t)$. Evaluating this as written would use 12 field additions (counting subtraction as addition), but a standard "fast Hadamard transform" reduces the 12 to 8.

Our representation of field elements for the Sandy Bridge (see Section 3) requires 6 limb additions for each field addition. There is no need to carry before the subsequent multiplications; this is the main reason that we use 6 limbs rather than 5 .

In a ladder step there are 4 copies of $H$, each requiring 8 field additions, each requiring 6 limb additions, for a total of 192 limb additions. This operation 
count suggests that 48 vector instructions suffice. Sandy Bridge has a helpful vaddsubpd instruction that computes $(a-e, b+f, c-g, d+h)$ given $(a, b, c, d)$ and $(e, f, g, h)$, obviously useful inside $H$.

However, we cannot simply vectorize across $x, y, z, t$. In Section 3 we were multiplying one $x$ by another, at the same time multiplying one $y$ by another, etc., with no permutations required; in this section we need to add $x$ to $y$, and this requires permutations.

The Sandy Bridge has a vector permutation unit acting in parallel with the adder and the multiplier, as noted in Section 3. But this does not mean that the cost of permutations can be ignored. A long sequence of permutations inside $H$ will force the adder and the multiplier to remain idle, since only a small fraction of the work inside $\mathbf{M}$ can begin before $H$ is complete.

Our original software used 48 vector additions and 144 vector permutations for the 4 copies of $H$. We then tackled the challenge of minimizing the number of permutations. We ended up reducing this number from 144 to just 36. This section presents the details; analyzes conditional swaps, which end up consuming further time in the permutation unit; and concludes by analyzing the total number of operations used in our Sandy Bridge software.

4.1. Limitations of the Sandy Bridge Permutations. There is a latency-1 permutation instruction vpermilpd that computes $(y, x, t, z)$ given $(x, y, z, t)$. vaddsubpd then produces $(x-y, y+x, z-t, t+z)$, which for the moment we abbreviate as $(e, f, g, h)$. At this point we seem to be halfway done: the desired output is simply $(f+h, f-h, e+g, e-g)$.

If we had $(f, h, e, g)$ at this point, rather than $(e, f, g, h)$, then we could apply vpermilpd and vaddsubpd again, obtaining $(f-h, h+f, e-g, g+e)$. One final vpermilpd would then produce the desired $(f+h, f-h, e+g, e-g)$. The remaining problem is the middle permutation of $(e, f, g, h)$ into $(f, h, e, g)$.

Unfortunately, Sandy Bridge has very few options for moving data between the left half of a vector, in this case $(e, f)$, and the right half of a vector, in this case $(g, h)$. There is a vperm $2 \mathrm{f} 128$ instruction (1-cycle throughput but latency 2 ) that produces $(g, h, e, f)$, but it cannot even produce $(h, g, f, e)$, never mind a combination such as $(f, h, e, g)$. (Haswell has more permutation instructions, but Ivy Bridge does not. This is not a surprising restriction: $n$-bit vector units are often designed as $n / 2$-bit vector units operating on the left half of a vector in one cycle and the right half in the next cycle, but this means that any communication between left and right requires careful attention in the circuitry. A similar leftright separation is even more obvious for the Cortex-A8.) We could shift some permutation work to the load/store unit, but this would have very little benefit, since simulating a typical permutation requires quite a few loads and stores.

The vpermilpd instruction $(x, y, z, t) \mapsto(y, x, t, z)$ mentioned above is one of a family of 16 vpermilpd instructions that produce ( $x$ or $y, x$ or $y, z$ or $t, z$ or $t$ ). There is an even more general family of 16 vshufpd instructions that produce ( $a$ or $b, x$ or $y, c$ or $d, z$ or $t$ ) given $(a, b, c, d)$ and $(x, y, z, t)$. In the first versions of our software we applied vshufpd to $(e, f, g, h)$ and $(g, h, e, f)$, obtaining $(f, h, g, e)$, and then applied vpermilpd to obtain $(f, h, e, g)$. 
Overall a single $H$ handled in this way uses, for each limb, 2 vaddsubpd instructions and 6 permutation instructions, half of which are handling the permutation of $(e, f, g, h)$ into $(f, h, e, g)$. The total for all limbs is 12 additions and 36 permutations, and the large "bubble" of permutations ends up forcing many idle cycles for the addition unit. This occurs four times in each ladder step.

4.2. Changing the Input/Output Format. There are two obvious sources of inefficiency in the computation described above. First, we need a final permutation to convert $(f-h, f+h, e-g, e+g)$ into $(f+h, f-h, e+g, e-g)$. Second, the middle permutation of $(e, f, g, h)$ into $(f, h, e, g)$ costs three permutation instructions, whereas $(g, h, e, f)$ would cost only one.

The first problem arises from a tension between Intel's vaddsubpd, which always subtracts in the first position, and the definition of $H$, which always adds in the first position. A simple way to resolve this tension is to store $(t, z, y, x)$ instead of $(x, y, z, t)$ for the input, and $\left(t^{\prime}, z^{\prime}, y^{\prime}, x^{\prime}\right)$ instead of $\left(x^{\prime}, y^{\prime}, z^{\prime}, t^{\prime}\right)$ for the output; the final permutation then naturally disappears. It is easy to adjust the other permutations accordingly, along with constants such as $\left(1, a^{2} / b^{2}, a^{2} / c^{2}, a^{2} / d^{2}\right)$.

However, this does nothing to address the second problem. Different permutations of $(x, y, z, t)$ as input and output end up requiring different middle permutations, but these middle permutations are never exactly the left-right swap provided by vperm $2 \mathrm{f} 128$.

We do better by generalizing the input/output format to allow negations. For example, if we start with $(x,-y, z, t)$, permute into $(-y, x, t, z)$, and apply vaddsubpd, we obtain $(x+y, x-y, z-t, t+z)$. Observe that this is not the same as the $(x-y, x+y, z-t, t+z)$ that we obtained earlier: the first two entries have been exchanged.

It turns out to be best to negate $z$, i.e., to start from $(x, y,-z, t)$. Then vpermilpd gives $(y, x, t,-z)$, and vaddsubpd gives $(x-y, x+y,-z-t, t-z)$, which we now abbreviate as $(e, f, g, h)$. Next vperm2f128 gives $(g, h, e, f)$, and independently vpermilpd gives $(f, e, h, g)$. Finally, vaddsubpd gives $(f-g, h+$ $e, h-e, f+g)$. This is exactly $\left(x^{\prime}, t^{\prime},-z^{\prime}, y^{\prime}\right)$ where $\left(x^{\prime}, y^{\prime}, z^{\prime}, t^{\prime}\right)=H(x, y, z, t)$.

The output format here is not the same as the input format: the positions of $t$ and $y$ have been exchanged. Fortunately, Figure 2.4 is partitioned by the $H$ rows into two separate universes, and there is no need for the universes to use the same format. We use the $(x, y,-z, t)$ format at the top and bottom, and the $(x, t,-z, y)$ format between the two $H$ rows. It is easy to see that exactly the same sequence of instructions works for all the copies of $H$, either producing $(x, y,-z, t)$ format from $(x, t,-z, y)$ format or vice versa.

$\mathbf{S}^{\mathbf{4}}$ and $\mathbf{M}^{\mathbf{4}}$ do not preserve negations: in effect, they switch from $(x, t,-z, y)$ format to $(x, t, z, y)$ format. This is not a big problem, since we can reinsert the negation at any moment using a single multiplication or low-latency logic instruction (floating-point numbers use a sign bit rather than twos-complement, so negation is simply xor with a 1 in the sign bit). Even better, in Figure 2.4(b), the problem disappears entirely: each $\mathbf{S}^{\mathbf{4}}$ and $\mathbf{M}^{\mathbf{4}}$ is followed immediately by a constant multiplication, and so we simply negate the appropriate constants. The resulting sequence of formats is summarized in Figure 4.3. 


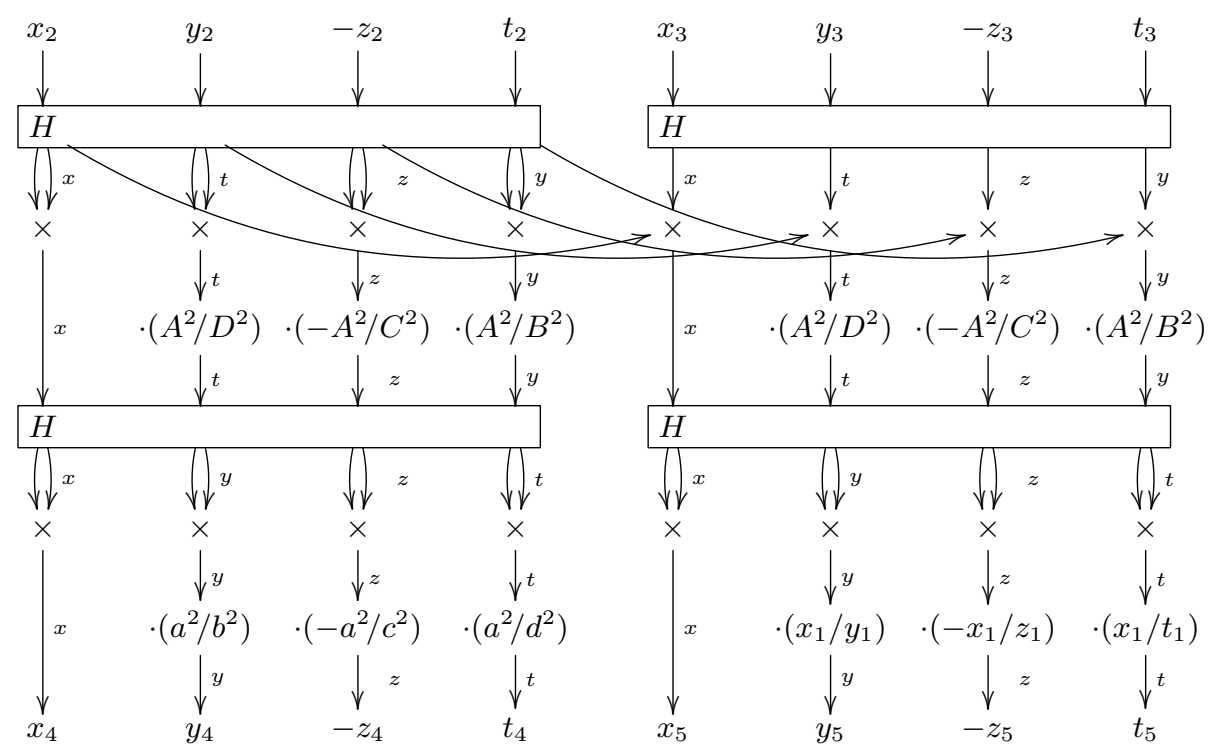

Fig. 4.3. Output format that we use for each operation in the right side of Figure 2.4 on Sandy Bridge, including permutations and negations to accelerate $H$

Each $H$ now costs 12 additions and just 18 permutations. The number of non-addition cycles that need to be overlapped with operations before and after $H$ has dropped from the original 24 to just 6 .

4.4. Exploiting Double Precision. We gain a further factor of 2 by temporarily converting from radix $2^{127 / 6}$ to radix $2^{127 / 3}$ during the computation of $H$. This means that, just before starting $H$, we replace the six limbs $\left(h_{0}, h_{1}, h_{2}, h_{3}, h_{4}, h_{5}\right)$ representing $h_{0}+h_{1}+h_{2}+h_{3}+h_{4}+h_{5}$ by three limbs $\left(h_{0}+h_{1}, h_{2}+h_{3}, h_{4}+h_{5}\right)$. These three sums, and the intermediate $H$ results, still fit into double-precision floating-point numbers.

It is essential to switch each output integer back to radix $2^{127 / 6}$ so that each output limb is small enough for the subsequent multiplication. Converting three limbs into six is slightly less expensive than three carries; in fact, converting from six to three and back to six uses exactly the same operations as three carries, although in a different order.

We further reduce the conversion cost by the following observation. Except for the $\mathbf{M}^{\mathbf{4}}$ by $\left(1: x_{1} / y_{1}: x_{1} / z_{1}: x_{1} / t_{1}\right)$, each of our multiplication results uses six carries, as explained in Section 3.4. However, if we are about to add $h_{0}$ to $h_{1}$ for input to $H$, then there is no reason to carry $h_{0} \rightarrow h_{1}$, so we simply skip that carry; we similarly skip $h_{2} \rightarrow h_{3}$ and $h_{4} \rightarrow h_{5}$. These skipped carries exactly cancel the conversion cost.

For the $\mathbf{M}^{\mathbf{4}}$ by $\left(1: x_{1} / y_{1}: x_{1} / z_{1}: x_{1} / t_{1}\right)$ the analysis is different: $h_{0}$ is large enough to affect $h_{2}$, and if we skipped carrying $h_{0} \rightarrow h_{1} \rightarrow h_{2}$ then the output 
of $H$ would no longer be safe as input to a subsequent multiplication. We thus carry $h_{0} \rightarrow h_{1}, h_{2} \rightarrow h_{3}$, and $h_{4} \rightarrow h_{5}$ in parallel; and then $h_{1} \rightarrow h_{2}, h_{3} \rightarrow h_{4}$, and $h_{5} \rightarrow h_{0}$ in parallel. In effect this $\mathbf{M}^{4}$ uses 9 carries, counting the cost of conversion, whereas in Section 3.4 it used only 8 .

To summarize, all of these conversions for all four $H$ cost just one extra carry, while reducing 48 additions and 72 permutations to 24 additions and 36 permutations.

4.5. Conditional Swaps. A ladder step starts from an input $(X(n P), X((n+$ 1) $P)$ ), which we abbreviate as $L(n)$, and produces $L(2 n)$ as output. Swapping the two halves of the input, applying the same ladder step, and swapping the two halves of the output produces $L(2 n+1)$ instead; one way to see this is to observe that $L(-n-1)$ is exactly the swap of $L(n)$.

Consequently one can reach $L(2 n+\epsilon)$ for $\epsilon \in\{0,1\}$ by starting from $L(n)$, conditionally swapping, applying the ladder step, and conditionally swapping again, where the condition bit is exactly $\epsilon$. A standard ladder reaches $L(n)$ by applying this idea recursively. A standard constant-time ladder reaches $L(n)$ by applying this idea for exactly $\ell$ steps, starting from $L(0)$, where $n$ is known in advance to be between 0 and $2^{\ell}-1$. An alternate approach is to first add to $n$ an appropriate multiple of the order of $P$, producing an integer known to be between (e.g.) $2^{\ell+1}$ and $2^{\ell+2}-1$, and then start from $L(1)$. We use a standard optimization, merging the conditional swap after a ladder step into the conditional swap before the next ladder step, so that there are just $\ell+1$ conditional swaps rather than $2 \ell$.

One way to conditionally swap field elements $x$ and $x^{\prime}$ using floating-point arithmetic is to replace $\left(x, x^{\prime}\right)$ with $\left(x+b\left(x^{\prime}-x\right), x^{\prime}-b\left(x^{\prime}-x\right)\right)$ where $b$ is the condition bit, either 0 or 1 . This takes three additions and one multiplication (times 6 limbs, times 4 field elements to swap). It is better to use logic instructions: replace each addition with xor, replace each multiplication with and, and replace $b$ with an all- 1 or all-0 mask computed from $b$. On the Sandy Bridge, logic instructions have low latency and are handled by the permutation unit, which is much less of a bottleneck for us than the addition unit.

We further improve the performance of the conditional swap as follows. The $\mathbf{M}^{4}$ on the right side of Figure 4.3 is multiplying $H$ of the left input by $H$ of the right input. This is commutative: it does not depend on whether the inputs are swapped. We therefore put the conditional swap after the first row of $H$ computations, and multiply the $H$ outputs directly, rather than multiplying the swap outputs. This trick has several minor effects and one important effect.

A minor advantage is that this trick removes all use of the right half of the swap output; i.e., it replaces the conditional swap with a conditional move. This reduces the original 24 logic instructions to just 18 .

Another minor advantage is as follows. The Sandy Bridge has a vectorized conditional-select instruction vblendvpd. This instruction occupies the permutation unit for 2 cycles, so it is no better than the 4 traditional logic instructions for a conditional swap: a conditional swap requires two conditional selects. However, this instruction is better than the 3 traditional logic instructions for a 
conditional move: a conditional move requires only one conditional select. This replaces the original logic instructions with 6 conditional-select instructions, consuming just 12 cycles.

A minor disadvantage is that the first $\mathbf{M}^{\mathbf{4}}$ and $\mathbf{S}^{\mathbf{4}}$ are no longer able to share precomputations of multiplications by $2^{-127}$. This costs us 3 multiplication instructions.

The important effect is that this trick reduces latency, allowing the $\mathbf{M}^{\mathbf{4}}$ to start much sooner. Adding this trick immediately produced a $5 \%$ reduction in our cycle counts.

\subsection{Total Operations. We treat Figure 2.4(b) as $2 \mathbf{M}^{\mathbf{4}}+3 \mathbf{S}^{\mathbf{4}}+3 \mathbf{m}^{\mathbf{4}}+4 H$.}

The main computations of $h_{i}$, not counting precomputations and carries, cost 30 additions and 36 multiplications for each $\mathbf{M}^{\mathbf{4}}, 15$ additions and 21 multiplications for each $\mathbf{S}^{\mathbf{4}}$, and 0 additions and 6 multiplications for each $\mathbf{m}^{\mathbf{4}}$. The total here is 105 additions and 153 multiplications.

The $\mathbf{M}^{\mathbf{4}}$ by $\left(1: x_{1} / y_{1}: x_{1} / z_{1}: x_{1} / t_{1}\right)$ allows precomputations outside the loop. The other $\mathbf{M}^{\mathbf{4}}$ consumes 5 multiplications for precomputations, and each $\mathbf{S}^{\mathbf{4}}$ consumes 8 multiplications for precomputations; the total here is 29 multiplications. We had originally saved a few multiplications by sharing precomputations between the first $\mathbf{S}^{\mathbf{4}}$ and the first $\mathbf{M}^{\mathbf{4}}$, but this is incompatible with the more important trick described in Section 4.5.

There are a total of 24 additions in the four $H$, as explained in Section 4.4. There are also 51 carries (counting the conversions described in Section 4.4 as carries), each consuming 3 additions and 2 multiplications, for a total of 153 additions and 102 multiplications.

The grand total is 282 additions and 284 multiplications, evidently requiring at least 284 cycles for each iteration of the main loop. Recall that there are various options to trade multiplications for additions: each $\mathbf{S}^{4}$ has 5 precomputed doublings that can each be converted from 1 multiplication to 1 addition, and each carry can be converted from 3 additions and 2 multiplications to 4 additions and 0 multiplications (or 4 additions and 1 multiplication for $h_{5} \rightarrow h_{0}$ ). We could use either of these options to eliminate one multiplication, reducing the 284-cycle lower bound to 283 cycles, but to reduce latency we ended up instead using the first option to eliminate 10 multiplications and the second option to eliminate 35 multiplications, obtaining a final total of 310 additions and 239 multiplications. These totals have been computer-verified.

We wrote functions in assembly for $\mathbf{M}^{\mathbf{4}}, \mathbf{S}^{\mathbf{4}}$, etc., but were still over 500 cycles. Given the Sandy Bridge floating-point latencies, and the requirement to keep two floating-point units constantly busy, we were already expecting instruction scheduling to be much more of an issue for this software than for typical integer-arithmetic software. We used various standard optimization techniques that were already used in several previous DH speed records: we merged the functions into a single loop, reorganized many computations to save registers, and eliminated many loads and stores. After building a new Sandy Bridge simulator and experimenting with different instruction schedules we ended up with our current loop, just 338 cycles, and a total of 88916 Sandy Bridge cycles 
for scalar multiplication. The main loop explains 84838 of these cycles; the remaining cycles are spent outside the ladder, mostly on converting $(x: y: z: t)$ to $(x / y: x / z: x / t)$ for output.

\section{Cortex-A8}

The low-power ARM Cortex-A8 core is the CPU core in the iPad 1, iPhone 4, Samsung Galaxy S, Motorola Droid X, Amazon Kindle 4, etc. Today a CortexA8 CPU, the Allwinner A10, costs just $\$ 5$ in bulk and is widely used in low-cost tablets, set-top boxes, etc. Like Sandy Bridge, Cortex-A8 is not the most recent microarchitecture, but its very wide deployment and use make it a sensible choice of platform for optimization and performance comparisons.

Bernstein and Schwabe in [15] (CHES 2012) analyzed the vector capabilities of the Cortex-A8 for various cryptographic primitives, and in particular set a new speed record for high-security DH, namely 460200 Cortex-A8 cycles. We do much better, just 274593 Cortex-A8 cycles, measured on a Freescale i.MX515. Our basic vectorization approach is the same for Cortex-A8 as for Sandy Bridge, and many techniques are reused, but there are also many differences. The rest of this section explains the details.

5.1. Cortex-A8 Vector Units. Each Cortex-A8 core has two 128-bit vector units operating in parallel on vectors of four 32-bit integers or two 64-bit integers:

- The arithmetic port takes one cycle for vector addition, with latency 2; or two cycles for vector multiplication (two 64-bit products $a c$, bd given 32-bit inputs $a, b$ and $c, d$ ), with latency 7 . Logic operations also use the arithmetic port.

- The load/store port handles loads, stores, and permutations. ARM's CortexA8 documentation [5] indicates that the load/store port can carry out one 128-bit load every cycle. Beware, however, that there are throughput limits on the L1 cache. We have found experimentally that the common TI Sitara Cortex-A8 CPU (used, e.g., in the Beaglebone Black development board) needs three cycles from one load until the next (this is what we call "Cortex-A8-slow"), while other Cortex-A8 CPUs ("Cortex-A8-fast") can handle seven consecutive cycles of loads without penalty.

There are three obvious reasons for Cortex-A 8 cycle counts to be much larger than Sandy Bridge cycle counts: registers are only 128 bits, not 256 bits; there are only 2 ports, not 6 ; and multiplication throughput is 1 every 2 cycles, not 1 every cycle. However, there are also speedups on Cortex-A8. There is (as in Haswell's floating-point units - see Appendix B online) a vector multiply-accumulate instruction with the same throughput as vector multiplication. A sequence of $m$ consecutive multiply-accumulate instructions that all accumulate into the same register executes in $2 m$ cycles (unlike Haswell), effectively reducing multiplication latency from 7 to 1 . Furthermore, Cortex-A8 multiplication produces 64-bit integer products, while Sandy Bridge gives only 53-bit-mantissa products. 
5.2. Representation. We decompose an integer $f$ modulo $2^{127}-1$ into five integer pieces in radix $2^{127 / 5}$ : i.e., we write $f$ as $f_{0}+2^{26} f_{1}+2^{51} f_{2}+2^{77} f_{3}+2^{102} f_{4}$. Compared to Sandy Bridge, having $20 \%$ more room in 64-bit integers than in 53-bit floating-point mantissas allows us to reduce the number of limbs from 6 to 5 . We require the small integers $f_{0}, f_{1}, f_{2}, f_{3}, f_{4}$ to be unsigned because this reduces carry cost from 4 integer instructions to 3 .

We arrange four integers $x, y, z, t$ modulo $2^{127}-1$ in five 128-bit vectors: $\left(x_{0}, y_{0}, x_{1}, y_{1}\right) ;\left(x_{2}, y_{2}, x_{3}, y_{3}\right) ;\left(x_{4}, y_{4}, z_{4}, t_{4}\right) ;\left(z_{0}, t_{0}, z_{1}, t_{1}\right) ;\left(z_{2}, t_{2}, z_{3}, t_{3}\right)$. This representation is designed to minimize permutations in $\mathbf{M}, \mathbf{S}$, and $H$. For example, computing $\left(x_{0}+z_{0}, y_{0}+t_{0}, x_{1}+z_{1}, y_{1}+t_{1}\right)$ takes just one addition without any permutations. The Cortex-A 8 multiplications take two pairs of inputs at a time, rather than four as on Sandy Bridge, so there is little motivation to put $\left(x_{0}, y_{0}, z_{0}, t_{0}\right)$ into a vector.

5.3. Optimizing M. Given an integer $f$ as above and an integer $g=g_{0}+$ $2^{26} g_{1}+2^{51} g_{2}+2^{77} g_{3}+2^{102} g_{4}$, the product $f g$ modulo $2^{127}-1$ is $h=h_{0}+$ $2^{26} h_{1}+2^{51} h_{2}+2^{77} h_{3}+2^{102} h_{4}$, with

$$
\begin{aligned}
& h_{0}=f_{0} g_{0}+2 f_{1} g_{4}+2 f_{2} g_{3}+2 f_{3} g_{2}+2 f_{4} g_{1}, \\
& h_{1}=f_{0} g_{1}+f_{1} g_{0}+f_{2} g_{4}+2 f_{3} g_{3}+f_{4} g_{2}, \\
& h_{2}=f_{0} g_{2}+2 f_{1} g_{1}+f_{2} g_{0}+2 f_{3} g_{4}+2 f_{4} g_{3}, \\
& h_{3}=f_{0} g_{3}+f_{1} g_{2}+f_{2} g_{1}+f_{3} g_{0}+f_{4} g_{4}, \\
& h_{4}=f_{0} g_{4}+2 f_{1} g_{3}+f_{2} g_{2}+2 f_{3} g_{1}+f_{4} g_{0} .
\end{aligned}
$$

There are 25 multiplications $f_{i} g_{j}$; additions are free as part of multiplyaccumulate instructions. We precompute $2 f_{1}, 2 f_{2}, 2 f_{3}, 2 f_{4}$ so that these values can be reused for another multiplication. These precomputations can be done by using either 4 shift or 4 addition instructions. Both shift and addition use 1 cycle per instruction, but addition has a lower latency. See Section 5.6 for the cost of carries.

5.4. Optimizing $\mathbf{S}$. The idea of optimizing $\mathbf{S}$ in Cortex-A8 is quite similar to Sandy Bridge; for details see Section 3.3. We state here only the operation count. Besides precomputation and carry, we use 15 multiplication instructions; some of those are actually multiply-accumulate instructions.

5.5. Optimizing $\mathbf{m}$. For $\mathbf{m}$ we compute only $h_{0}=c f_{0}, h_{1}=c f_{1}, h_{2}=c f_{2}$, $h_{3}=c f_{3}$, and $h_{4}=c f_{4}$, again exploiting the small constants stated in Section 2.6.

Recall that we use unsigned representation. We always multiply absolute values, then negate results as necessary by subtracting from $2^{129}-4: n_{0}=$ $2^{28}-4-h_{0}, n_{1}=2^{27}-4-h_{1}, n_{2}=2^{28}-4-h_{2}, n_{3}=2^{27}-4-h_{3}, n_{4}=2^{27}-4-h_{4}$.

Negating any subsequence of $x, y, z, t$ costs at most 5 vector subtractions. Negating only $x$ or $y$, or both $x$ and $y$, costs only 3 subtractions, because our representation keeps $x, y$ within 3 vectors. The same comment applies to $z$ and $t$. The specific $\mathbf{m}$ in Section 2.6 end up requiring a total of 13 subtractions with the same cost as 13 additions. 
5.6. Carries. Each multiplication uses at worst 6 serial carries $h_{1} \rightarrow h_{2} \rightarrow h_{3} \rightarrow$ $h_{4} \rightarrow h_{0} \rightarrow h_{1}$, each costing 3 additions. Various carries are eliminated by the ideas of Section 3.4.

5.7. Hadamard Transform. See Appendix A online.

5.8. Total Arithmetic. We view Figure $2.4(\mathrm{~b})$ as $4 \mathbf{M}^{2}+6 \mathbf{S}^{\mathbf{2}}+6 \mathbf{m}^{\mathbf{2}}+4 H$. Here we combine $x$ multiplications and $y$ multiplications into a vectorized $\mathbf{M}^{\mathbf{2}}$, and similarly combine $z$ multiplications and $t$ multiplications; this fits well with the Cortex-A8 vector multiplication instruction, which outputs two products.

The main computations of $h_{i}$, not counting precomputations and carries, cost 0 additions and 25 multiplications for each $\mathbf{M}, 0$ additions and 15 multiplications for each $\mathbf{S}, 0$ additions and 5 multiplications for each $\mathbf{m}$, and 15 additions for each $H$ block. The total here is 60 additions and 220 multiplications.

Each $\mathbf{M}$ costs 4 additions for precomputations, and each $\mathbf{S}$ also costs 4 additions for precomputations. Some precomputations can be reused. The cost of precomputations is 20 additions.

There are 10 carry blocks using 6 carries each, and 6 carry blocks using 5 carries each. Each carry consists of 1 shift, 1 addition, and 1 logical and. This cost is equivalent to 3 additions. There are another 13 additions needed to handle negation. Overall the carries cost 283 additions. Two conditional swaps, each costing 9 additions, sum up to 18 additions.

In total we have 381 additions and 220 multiplications in our inner loop. This means that the inner loop takes at least 821 cycles.

We scheduled instructions carefully but ended up with some overhead beyond arithmetic: even though the arithmetic and the load/store unit can operate in parallel, latencies and the limited number of registers leave the arithmetic unit idle for some cycles. Sobole's simulator at [48], which we found very helpful, reports 966 cycles. Actual measurements report 986 cycles; the 251 ladder steps thus account for 247486 of our 273349 cycles.

\section{References}

[5] ARM Limited: Cortex-A8 technical reference manual, revision r3p2 (2010), http://infocenter.arm.com/help/topic/com.arm.doc.ddi0344k/DDI0344K_ cortex_a8_r3p2_trm.pdf

[8] Bernstein, D.J.: Floating-point arithmetic and message authentication (2004), http://cr.yp.to/papers.html\#hash127

[9] Bernstein, D.J.: Curve25519: new Diffie-Hellman speed records. In: PKC 2006. LNCS, vol. 3958, pp. 207-228 (2006)

[10] Bernstein, D.J.: Elliptic vs. hyperelliptic, part 1 (2006), http://cr .yp.to/talks . html\#2006.09.20

[11] Bernstein, D.J., Duif, N., Lange, T., Schwabe, P., Yang, B.-Y.: High-speed highsecurity signatures. In: CHES 2011. LNCS, vol. 6917 (2011); see also newer version $[12]$

[12] Bernstein, D.J., Duif, N., Lange, T., Schwabe, P., Yang, B.-Y.: High-speed highsecurity signatures. Journal of Cryptographic Engineering 2, 77-89 (2012); see also older version [11] 
[13] Bernstein, D.J., Lange, T. (eds.): eBACS: ECRYPT Benchmarking of Cryptographic Systems, accessed 25 September 2014 (2014), http://bench.cr.yp.to

[15] Bernstein, D.J., Schwabe, P.: NEON crypto. In: CHES 2012. LNCS, vol. 7428, pp. 320-339 (2012)

[17] Bos, J.W., Costello, C., Hisil, H., Lauter, K.: Fast cryptography in genus 2. In: Eurocrypt 2013. LNCS, vol. 7881, pp. 194-210 (2013)

[18] Bos, J.W., Costello, C., Hisil, H., Lauter, K.: High-performance scalar multiplication using 8-dimensional GLV/GLS decomposition. In: CHES 2013. LNCS, vol. 8086, pp. 331-348 (2013)

[21] Chudnovsky, D.V., Chudnovsky, G.V.: Sequences of numbers generated by addition in formal groups and new primality and factorization tests. Advances in Applied Mathematics 7, 385-434 (1986)

[22] Costello, C., Hisil, H., Smith, B.: Faster compact Diffie-Hellman: endomorphisms on the x-line. In: Eurocrypt 2014. LNCS, vol. 8441, pp. 183-200 (2014)

[23] Costigan, N., Schwabe, P.: Fast elliptic-curve cryptography on the Cell Broadband Engine. In: Africacrypt 2009. LNCS, vol. 5580, pp. 368-385 (2009)

[24] Faz-Hernández, A., Longa, P., Sánchez, A.H.: Efficient and secure algorithms for GLV-based scalar multiplication and their implementation on GLV-GLS curves. In: CT-RSA 2014. LNCS, vol. 8366, pp. 1-27 (2013)

[25] Fog, A.: Instruction tables: Lists of instruction latencies, throughputs and microoperation breakdowns for Intel, AMD and VIA CPUs (2014), http://agner.org/ optimize/

[26] Galbraith, S., Lin, X., Scott, M.: Endomorphisms for faster elliptic curve cryptography on a large class of curves. In: Eurocrypt 2009. LNCS, vol. 5479, pp. 518-535 (2009)

[27] Gallant, R.P., Lambert, R.J., Vanstone, S.A.: Faster point multiplication on elliptic curves with efficient endomorphisms. In: Crypto 2001. LNCS, vol. 2139, pp. 190-200 (2001)

[30] Gaudry, P.: Variants of the Montgomery form based on Theta functions (2006); see also newer version [31], http://www. loria.fr/ gaudry/publis/toronto.pdf

[31] Gaudry, P.: Fast genus 2 arithmetic based on Theta functions. Journal of Mathematical Cryptology 1, 243-265 (2007); see also older version [30]

[32] Gaudry, P., Lubicz, D.: The arithmetic of characteristic 2 Kummer surfaces and of elliptic Kummer lines. Finite Fields and Their Applications 15, 246-260 (2009)

[33] Gaudry, P., Schost, É: Genus 2 point counting over prime fields. Journal of Symbolic Computation 47, 368-400 (2012)

[34] Hamburg, M.: Fast and compact elliptic-curve cryptography (2012), https:// eprint.iacr.org/2012/309

[40] Longa, P., Sica, F.: Four-dimensional Gallant-Lambert-Vanstone scalar multiplication. In: Asiacrypt 2012. LNCS, vol. 7658, pp. 718-739 (2012)

[42] Montgomery, P.L.: Speeding the Pollard and elliptic curve methods of factorization. Mathematics of Computation 48, 243-264 (1987)

[44] Oliveira, T., López, J., Aranha, D.F., Rodríguez-Henríquez, F.: Lambda coordinates for binary elliptic curves. In: CHES 2013. LNCS, vol. 8086, pp. 311-330 (2013)

[48] Sobole, É.: Calculateur de cycle pour le Cortex A8 (2012), http://pulsar. webshaker.net/ccc/index.php 